\title{
Evolution of the Mass Loss Rate During Atmospheric and Pressurized Slow Pyrolysis of Wheat Straw in a Bench-Scale Reactor
}

\author{
Gianluca Greco, María Videgain, Belén González, Joan J. Manyà \\ Biochar Research Lab, Thermochemical Processes Research Group (GPT), \\ Aragón Institute of Engineering Research (I3A), \\ Technological College of Huesca, University of Zaragoza, Spain. \\ Tel. +34-974239320, e-mail:: greco@unizar.es
}

\begin{abstract}
A deep study focused on the significant effect of the absolute pressure on the yield of produced gas during the slow pyrolysis of biomass was carried out. In addition, the evolution of the mass loss rate linked to the pyrolysis process was also analyzed.
\end{abstract}

\section{Introduction}

Biomass has been receiving considerable interest as a renewable energy source in recent years, due to the worldwide incresead concerns about the climate change, enviromental pollution and the fossil fuels depletion. A traditional way to produce charcoal and gas starting from biomass is the slow pyrolysis process. In this study, the absolute pressure effect on the yields of the pyrolysis products as well as the evolution of mass loss rate linked to the process was analyzed. An increase in pressure can result in an increased production of total produced gas, probably due to an enhancement of the secondary reactions of tar vapors. An increased pressure can also lead to higher cumulative yields $\mathrm{ofCH}_{4}$, as a consequence of the promotion of the methanation reactions. To better understand the pyrolysis behaviour in a bench-scale fixed-bed reactor, it can be very useful to measure the real time mass loss rate. The acquisition of the real time simple weight in such systems can allow reasearchers to study termal processes involving mass and heat transfer limitations [1]. The specific aim of this study is to determine the effect of the absolute pressure (at 0.1 $\mathrm{MPa}$ and at $0.5 \mathrm{MPa}$ ), at a constant peak temperature of $500{ }^{\circ} \mathrm{C}$, on the pyrolysis behavior of wheat straw pellets in a fixed-bed reactor equipped with a weighing platform. Several blank tests have been performed as a preliminary step in order to take into account the presence of the buoyancy effect due to the significant changes in the density of the surrounding air. After subtracting the signal from blank tests, the net mass loss data was studied, combined with the data concerning the gas composition provided by a micro-GC, as well as the dynamic temperature profiles measured by four thermocouples placed at different heights in the reactor.

\section{Experimental}

\section{Materials and experimental conditions}

A type of wheat straw pellets provided by Ghent University (Belgium) has been selected as feedstock for the experiments. The experimental conditions chosen for the set of experiments are visible in Table 1 . The variable parameters are the absolute pressure $(0.1 \mathrm{MPa}$ and $0.5 \mathrm{MPa})$ and the reactor atmosphere $\left(100 \% \mathrm{~N}_{2}, 60 \% \quad \mathrm{CO}_{2}\right.$ and $40 \% \mathrm{~N}_{2}$, respectively), by keeping constant the peak temperature $\left(500{ }^{\circ} \mathrm{C}\right)$ and the residence time of the gas phase within the reactor (100 s). The soaking time (i.e., the time elapsed at the peak temperature) was always kept at 1 hour for every single experiment.

\section{Pyrolysis device and procedures}

The experimental device is based on a cylindrical and vertical reactor made of stainless steel and electrically heated. A basket of $4 \mathrm{~L}$, was used to allocate the biomass into the reactor. The reactor was supported on a ceramic tube, which was placed on a weighing platform. More details regarding the configuration of the reactor is available in a previous publication [2]. The composition of the gas fraction $\left(\mathrm{N}_{2}\right.$, $\mathrm{CO}_{2}, \mathrm{CH}_{4}, \mathrm{CO}, \mathrm{C}_{2} \mathrm{H}_{\mathrm{X}}$ and $\mathrm{H}_{2}$ ) was determined using a dual channel micro-GC (Agilent 490).

\section{Results}

As a first step, the weight loss profiles of the biomass was investigated. In order to have a higher 
reproducibility of the results and to assess the buoyancy effect, the raw signal of every single experiment was subtracted with a blank signal, previously determined from the corresponding blank test. It was expected to observe an experimental difference in weight very close to the actual one, but the results are not consistent so far, showing a low level of reproducibility. This is probably due to the high sensitivity of the weighing platform: even a small difference in the reactor position inside the furnace, as well as a difference in the heating programme, could lead to very different results in terms of weight. Examples of the results obtained after such experiments are visible in Figures 1 and 2 . The first one shows the weight variation and the temperature profiles along the time. The trend presents a soft decrease in weight at $100{ }^{\circ} \mathrm{C}$ due to the water content evaporation of the biomass. Then, a sharp loss in weight has been recorded when the biomass devolatilization occurred. Such reactions start between $150{ }^{\circ} \mathrm{C}$ and $200{ }^{\circ} \mathrm{C}$, depending on the system pressure. After this step, the weight variation tends to stabilize to a steady value. In Figure 2 a comparison between the weight profiles of different experiments carried out at $0.1 \mathrm{MPa}$ and $0.5 \mathrm{MPa}$ is displayed. As it can be seen, an increase in pressure led to a faster weight loss of the biomass, probably due to an enhancement of the reactions kinetics as well as a slight shift of the devolatilization beginning towards lower temperatures. From a gas yield point of view, the higher the pressure, the higher the total amount of gases produced; conversely, a decrease in the liquid yield by increasing the pressure has been displayed. Furthermore, it seems that the char yield is not influenced by changes in pressure.

\section{Conclusions}

The effects of the absolute pressure on the slow pyrolysis process have been analysed, in terms of mass loss profiles and product yields. Further investigations about the buoyancy effect are needed, since the net weight loss profiles (i.e. obtained by subtracting the experimental raw signal with a blank profile) have not given consistent results so far, showing a quite low reproducibility.

\section{Acknowledgements}

This project has received funding from the European Union's Horizon 2020 research and innovation programme under the Marie Sklodowska-Curie grant agreement No 721991.

\section{References}

[1] D. Tapasvi, D. Khalil, O. Skreiberg, K.-Q. Tran, and M. Gronli, "Torrefaction of Norwegian Birch and Spruce; an Experimental Study Using Macro-TGA," Energy \& Fuels, vol. 26, pp. 5232-5240, 2012.

[2] J. J. Manyà, D. Alvira, M. Azuara, D. Bernin, and N. Hedin, "Effects of Pressure and the Addition of a Rejected Material from Municipal Waste Composting on the Pyrolysis of Two-Phase Olive Mill Waste," Energy \& Fuels, vol. 30, pp. 8055-8064, 2016. 


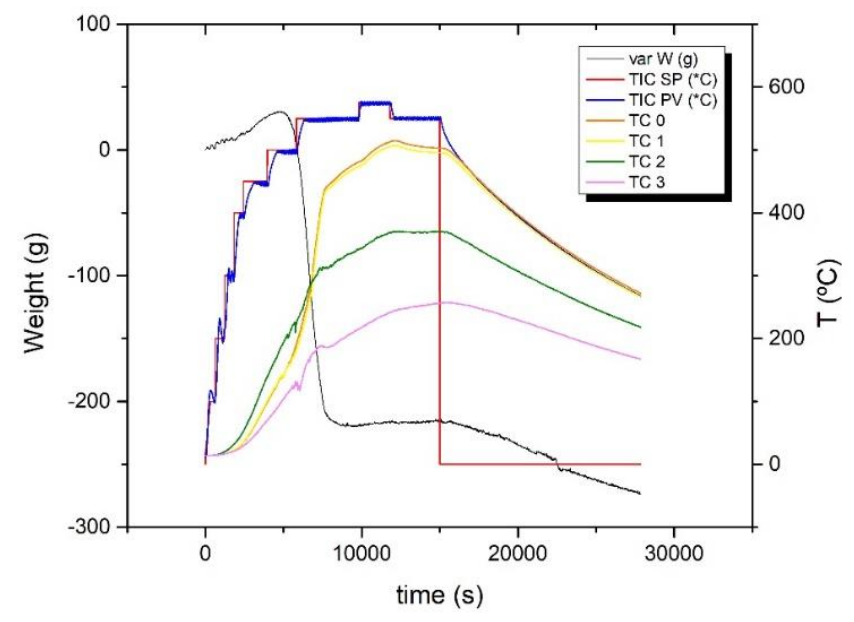

Figure 1: Set of experiments

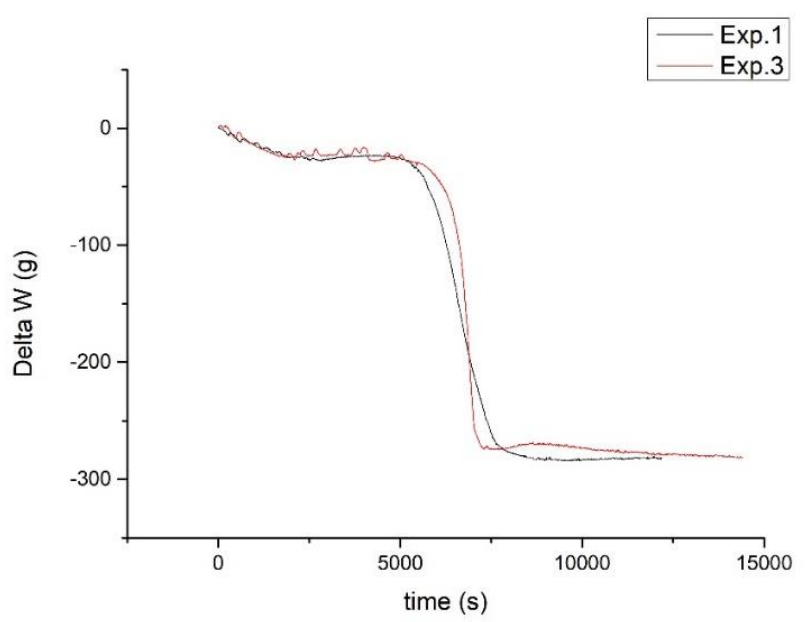

Figure 2: Weight loss comparison between Experiment 1 and Experiment 3

Table 1: Set of experiments carried out.

$\begin{array}{cccccc}\text { run } & T_{p}\left({ }^{\circ} \mathrm{C}\right) & \mathrm{P}(\mathrm{MPa}) & \mathrm{t}(\mathrm{s}) & \% N_{2} & \% \mathrm{CO}_{2} \\ 1 & 500 & 0.1 & 100 & 100 & 0 \\ 2 & 500 & 0.1 & 100 & 40 & 60 \\ 3 & 500 & 0.5 & 100 & 100 & 0 \\ 4 & 500 & 0.5 & 100 & 40 & 60\end{array}$

